\title{
Video Article \\ Imaging G Protein-coupled Receptor-mediated Chemotaxis and its Signaling Events in Neutrophil-like HL60 Cells
}

\author{
Xi Wen ${ }^{1}$, Tian $\operatorname{Jin}^{1}$, Xuehua Xu ${ }^{1}$ \\ ${ }^{1}$ Chemotaxis Signal Section, Laboratory of Immunogenetics, National Institute of Allergy and Infectious Diseases, National Institutes of Health
}

Correspondence to: Xuehua Xu at XXU@niaid.nih.gov

URL: https://www.jove.com/video/54511

DOI: doi:10.3791/54511

Keywords: Cellular Biology, Issue 115, Neutrophil chemotaxis, G protein-coupled receptor (GPCR), G-proteins, signal transduction, cell migration

Date Published: 9/14/2016

Citation: Wen, X., Jin, T., Xu, X. Imaging G Protein-coupled Receptor-mediated Chemotaxis and its Signaling Events in Neutrophil-like HL60 Cells. J. Vis. Exp. (115), e54511, doi:10.3791/54511 (2016).

\section{Abstract}

Eukaryotic cells sense and move towards a chemoattractant gradient, a cellular process referred as chemotaxis. Chemotaxis plays critical roles in many physiological processes, such as embryogenesis, neuron patterning, metastasis of cancer cells, recruitment of neutrophils to sites of inflammation, and the development of the model organism Dictyostelium discoideum. Eukaryotic cells sense chemo-attractants using $\mathrm{G}$ proteincoupled receptors. Visual chemotaxis assays are essential for a better understanding of how eukaryotic cells control chemoattractant-mediated directional cell migration. Here, we describe detailed methods for: 1) real-time, high-resolution monitoring of multiple chemotaxis assays, and 2) simultaneously visualizing the chemoattractant gradient and the spatiotemporal dynamics of signaling events in neutrophil-like HL60 cells.

\section{Video Link}

The video component of this article can be found at https://www.jove.com/video/54511/

\section{Introduction}

Eukaryotic cells sense and move toward the higher concentration within a chemoattractant gradient, a cellular process referred as chemotaxis. Chemotaxis plays critical roles in many physiological processes, such as embryogenesis ${ }^{1}$, neuron patterning ${ }^{2}$, metastasis of cancer cells ${ }^{3}$, recruitment of neutrophils to sites of inflammation ${ }^{4}$, and the development of the model organism Dictyostelium discoideum ${ }^{5}$. In general, eukaryotic cells sense chemoattractants using $\mathrm{G}$ protein-coupled receptors ${ }^{5}$. The engagement of chemoattractants with these receptors promotes dissociation of the heterotrimeric $G$ proteins $G \alpha$ and $G \beta y$, which in turn activate downstream signal transduction pathways that ultimately regulate the spatiotemporal organization of the actin cytoskeleton to drive cell migration ${ }^{5-9}$.

Cell biologists have been developing and improving chemotaxis assays to examine how G protein-coupled receptor (GPCR) signaling mediates directed cell migration. The Boyden chamber or transwell migration assay was developed in 1960 by Boyden ${ }^{10}$. The assay works by creating a gradient of chemoattractant compounds between two wells that are separated by a microporous membrane. Its simplicity and ease of use make it the most widely used chemotaxis assay to date. However, the assay does not enable the migrating process of the cells to be visualized. The Zigmond chamber is the first visual microfluidic device that allows clear imaging of cell migration on a coverslip across a narrow constriction toward a source chemoattractant ${ }^{11}$. Dunn ${ }^{12}$ and Insall ${ }^{13}$ modified and improved the high-resolution and long-term imaging capability of the Zigmond chamber chemotaxis assay. Because of the highly predictable, diffusion-dominant characteristics of fluid flow, microfluidics has been providing solutions for next-generation chemotaxis assays such as EZ-TAXIScan (a cell mobility analysis device).

With the stability of the gradient ensured, the device allows six chemotaxis assays to be carried out simultaneously (Figure 1A). In contrast to the directionally fixed gradients generated in the various chamber assays above, the needle or micropipette assay developed by Guenther Gerisch generates a gradient with a movable source ${ }^{14}$. In the assay, chemoattractant is released from a movable micropipette to generate a stable gradient. With this needle assay, researchers found that different cells generate pseudopods with fundamentally different characteristics. Applying fluorescent microscopy, we were able to visualize the gradient to facilitate its quantitative measurement throughout ${ }^{15}$. In this study, we describe detailed methods for preparing chemotactic HL60 (human promyelocytic leukemia) cells, simultaneously monitoring multiple chemotaxis assays with the cell mobility analysis device, and visualizing the GPCR-mediated spatiotemporal dynamics of signaling molecules such as protein kinase D1 in single live cells in response to visible, spatiotemporally controllable chemoattractant stimuli. Our advanced imaging methods can be applied to general chemotaxis studies, and are especially suitable for mammalian cell systems. 


\section{Culture and Differentiation of Human Neutrophil-like HL60 Cells}

1. Prepare RPMI (Roswell Park Memorial Institute) 1640 culture medium which contains RPMI 1640 medium, $10 \%$ ( $/ v$ ) fetal bovine serum (FBS), $0.1 \mathrm{mM}$ sodium pyruvate, and $25 \mathrm{mM}$ HEPES (4-(2-hydroxyethyl)-1-piperazineethanesulfonic acid). Warm the RPMI 1640 culture medium to $37^{\circ} \mathrm{C}$

2. Using a hemocytometer determine the cell density of HL60 cells to make sure the cells are in the log-phase growth stage. NOTE: Cells are usually in log phase on the third day after passaging. The density of log-phase cells is usually $6-8 \times 10^{5}$ cells $/ \mathrm{ml}$. The cell density required to start a new culture is about $1.5 \times 10^{5}$ cells $/ \mathrm{ml}$.

NOTE: For example, for a $10 \mathrm{ml}$ new culture in a T-75 flat flask, if the cell density of log-phase cells is $6 \times 10^{5}$ cells $/ \mathrm{ml}$, then $2.5 \mathrm{ml}$ of logphase cells $\left(6 \times 10^{5}\right)$ is diluted to $1.5 \times 10^{5}$ cells $/ \mathrm{ml} \mathrm{in} 10 \mathrm{ml}$ new culture. To make a final volume of $10 \mathrm{ml}$ culture, $7.5 \mathrm{ml}$ of RPMl $1640 \mathrm{culture}$ medium is added.

3. Place the calculated volume of warm RPMI 1640 culture medium into a flat flask. For a T-75 flat flask culture, the total volume of the cell culture is $10 \mathrm{ml}$.

4. Add the calculated volume of log-phase growing HL60 cells into the flat flask to reach a cell density of $1.5 \times 10^{5} \mathrm{cells} / \mathrm{ml}$.

5. Incubate the cells at $37{ }^{\circ} \mathrm{C}$ in a humidified incubator with $5 \% \mathrm{CO}_{2}$. NOTE: The doubling time of HL60 is almost $24 \mathrm{hr}$.

6. Passage the cells with RPMI 1640 culture medium every $2-3$ days. It is important that the cell density of the HL60 cells never exceeds $1.5 x$ $10^{6} \mathrm{cells} / \mathrm{ml}$ and the cell passage lasts no longer than 2 months.

7. Differentiate the HL60 cells 5 days before the experiments.

NOTE: RPMI 1640 differentiation medium contains RPMI 1640 medium, 10\% (v/v) fetal bovine serum (FBS), 0.1 mM sodium pyruvate, 25 mM HEPES, and 1.3\% dimethyl sulfoxide (DMSO). In other words, it is $1.3 \%$ DMSO in RPMI 1640 culture medium.

1. To differentiate HL60 cells, pre-warm the RPMI 1640 culture medium to $37^{\circ} \mathrm{C}$. Add the warm RPMI 1640 culture medium into a flat flask.

2. For a final $10 \mathrm{ml} \mathrm{HL60} \mathrm{differentiation} \mathrm{culture,} \mathrm{add} 130$ ul DMSO directly to the RPMI 1640 culture medium in the flat flask while swirling the flask so that the DMSO is rapidly diluted by the RPMI 1640 culture medium.

3. Add log-phase HL60 cells to a final cell density of $1.5 \times 10^{5}$ cells $/ \mathrm{ml}$ in a final $10 \mathrm{ml} \mathrm{RPMI} 1640$ differentiation medium. Culture the HL60 cells in RPMI 1640 differentiation medium at $37^{\circ} \mathrm{C}$ in a humidified incubator with $5 \% \mathrm{CO}_{2}$ for 5 days.

\section{Coating the Cover Glass Surface of the 4 well Chamber}

1. Take the bottle of $2 \%$ gelatin stock solution from the refrigerator, clean it with $70 \%$ ethanol, and place it in the hood. Take $1 \mathrm{ml} 2 \%$ gelatin stock solution and return the remaining gelatin stock solution to the refrigerator immediately.

2. Allow the gelatin solution to completely liquefy at $37^{\circ} \mathrm{C}$ and pipette it thoroughly. Dilute the $2 \%$ gelatin stock solution with $9 \mathrm{ml}$ pre-warmed $1 \mathrm{x}$ Hanks' balanced salt solution (HBSS) buffer to a final concentration of $0.2 \%$ gelatin.

3. Add $0.5 \mathrm{ml}$ or $2 \mathrm{ml} 0.2 \%$ gelatin in HBSS to the two middle wells of a 4-well chamber or a 1-well chamber with a cover glass in the bottom, respectively. Tilt the plates in several directions so that the liquid covers the entire surface area.

4. Place the plates in a $37^{\circ} \mathrm{C}$ incubator. The plates will be ready for use in $1 \mathrm{hr}$. They can be used for up to 5 days. Just before seeding cells, remove the gelatin solution from the 4 -well or 1 -well chambers.

\section{Chemotaxis Assay Using Cell Mobility Analysis Device}

1. Prepare and warm RPMI 1640 starving medium, which is RPMI medium containing $0.1 \mathrm{mM}$ sodium pyruvate (optional) and $25 \mathrm{mM}$ HEPES.

2. Prepare $100 \mu \mathrm{l}$ of $100 \mathrm{nM}$ fMLP (N-formylmethionyl-leucyl-phenylalanine) in RPMI 1640 starving medium.

3. Prepare $3 \mathrm{ml}$ of $1 \%$ bovine serum albumin (BSA)/RPMI 1640 medium which contains $1 \%$ BSA in RPMl 1640 starving medium and $10 \mathrm{ml}$ of $0.1 \%$ BSA/RPMI 1640 medium.

4. Coat a $22 \mathrm{~mm}$ square coverslip and a $5 \mu \mathrm{m}$ cell mobility analysis device chip with freshly prepared $1 \% \mathrm{BSA} / \mathrm{RPMI} 1640 \mathrm{medium}$ for $1 \mathrm{hr}$ at RT.

5. Assemble the Holder Using the Following Procedure:

1. Position the holder base with the levers in the vertical position so that the levers can tilt toward the user. Apply $70 \%$ ethanol solution to the surface of the $41 \mathrm{~mm}$ glass. Carefully wipe the surfaces with a wipe, taking care not to scratch them.

2. Insert the $41 \mathrm{~mm}$ glass into the holder base. Put the BSA-coated $22 \mathrm{~mm}$ square coverslip on top of the $41 \mathrm{~mm}$ glass in the holder base. NOTE: The coated square coverslip between the $41 \mathrm{~mm}$ glass and the chip allows chemotaxis to occur on the substratum of the coating surface.

3. Insert the O-ring (small) into the bottom of the wafer housing, and mount the wafer housing into the holder base with the elliptical hole at the rear. Pull the inner level of the holder base forward to the horizontal position to lock the wafer housing in position.

4. Blow any dust from the interior of the wafer housing with an air duster. Add $4 \mathrm{ml}$ of $0.1 \% \mathrm{BSA} / \mathrm{RPMI} 1640$ medium into the wafer housing.

5. Mount the BSA-coated cell mobility analysis device chip in the center of the wafer housing with the structural face downwards in contact with the glass.

NOTE: That the chip needs to be inserted with the positioning mark (the hole in the top of the chip) at the rear.

6. Affix the rubber gasket to the bottom of the wafer clamp by fitting the two protrusions on the rubber gasket into the holes.

7. Mount the O-ring (large) onto the top of the wafer housing. Mount the wafer clamp with the sensor hole at the rear. Pull the outer lever of the housing base forward to the horizontal position to lock the wafer clamp in place. 
8. After assembling the holder, place the bottom of the holder on the light box to confirm that there are no air bubbles in the wells. If air bubbles are detected, remove them using the provided plastic syringe fitted with a sample loading tip.

9. Remove the cover and put the assembly onto the plate on top of the cell mobility analysis device unit. Attach the sensor block to the holder.

6. Prepare HL60 Cells for Chemotaxis Assay:

1. Calculate the cell density of the differentiated HL60 cells with a hemocytometer. After 5 days of differentiation, the cell density is around $1.0 \times 10^{6} \mathrm{cell} / \mathrm{s} / \mathrm{ml}$.

2. Calculate the final volume of HL60 cells at a final cell density of $2 \times 10^{6}$ cells $/ \mathrm{ml}$. For example, if the cell density of the differentiated HL60 cells is $1.0 \times 10^{6} \mathrm{cells} / \mathrm{ml}$, then $10 \mathrm{ml}$ of differentiated HL60 cells will be resuspended with $5 \mathrm{ml}$ of $0.1 \%$ BSA/RPMI 1640 medium to reach $2 \times 10^{6} \mathrm{cells} / \mathrm{ml}$.

3. Centrifuge differentiated HL60 cells at $200 \times \mathrm{g}$ for $5 \mathrm{~min}$ at RT. Remove the supernatant and resuspend the cells with the calculated volume of $0.1 \%$ BSA/RPMI 1640 medium at step 3.6.2) to a final cell density of $2 \times 10^{6} \mathrm{cells} / \mathrm{ml}$.

7. Start up the cell mobility analysis device unit and PC for image acquisition. Connect the unit to the PC. Turn on the cell mobility analysis device. Turn on the PC.

8. Start Up the Cell Mobility Analysis Device Software.

1. Observe 5 control panels: camera, heater, shooting, memo, and camera images.

2. On the camera image control panel, use "Horizontal line" or "Vertical line" to adjust the holder/view position in real time to center the camera in the camera image panel.

3. On the camera control panel, select $\mathrm{CH} 1$ to $\mathrm{CH} 6$ to move the camera to the defined channel. To center the view fields of a channel, click "Move L" or "Move R" to adjust the X-coordinate of the camera and center the camera's position horizontally.

4. Adjust the Y-coordinate of the camera by turning the position knob on the front panel of the cell mobility analysis device unit to vertically adjust the image to center the screen.

5. On the heater control panel, set the "holder temp" to $37.0^{\circ} \mathrm{C}$ and the "plate temp" to $39^{\circ} \mathrm{C}$. Click "Heat" to start heating. Also click "holder" to control the temperature using the thermal sensor connected to the holder.

6. On the shooting panel, enter "15 sec" at "Interval" and "30 min" at "Time" for the intervals and the duration of the chemotaxis assay. Check "Heater off at the end of shooting" for safety reasons. Designate a destination for the stored images.

7. On the memo panel, input the specifics of the experiments, such as cell line used, whether or not treatment was applied, and the concentration of chemo-attractants applied.

8. Confirm the center position of all of the channels that will be used in the experiments, and repeat the adjustment for all channels if needed.

9. Inject and Align Cells as Follows:

1. Remove the buffer from the holder, and take out $8 \mu$ l of buffer from the third well from the top for each channel.

2. Use a syringe to inject $2 \mu$ of cells into the second well in the same channel while monitoring the screen in real time to control cell number and flow during injection. After the cells are nicely aligned, immediately add the $8 \mu \mathrm{l}$ of buffer back. Repeat the same procedure for the other channels (Figure 1B).

10. Add $2 \mathrm{ml}$ buffer back to the holder, and add $1 \mu \mathrm{l} 100 \mathrm{nM}$ fMLP to the third well from the top. Start image acquisition and save the data. Analyze the data obtained using image tracing software $^{9}$ (Figure 2).

\section{Transfection with Electroporation}

1. Differentiate HL60 cells 5 days before the experiment as described in Section 1. Coat 4-well or 1-well chambers as instructed in Section 2.

2. Prepare and warm RPMI 1640 electroporation recovery medium, which contains RPMI 1640 medium, $20 \%$ (v/v) FBS, 0.1 mM sodium pyruvate, and $25 \mathrm{mM}$ HEPES, to $37^{\circ} \mathrm{C}$.

3. Right before starting the transfection experiment, add either $300 \mu \mathrm{l}$ or $3 \mathrm{ml}$ RPMl 1640 culture medium into the wells of the pre-coated 4 -well or 1-well chambers, respectively.

4. Incubate the chambers in a humidified $37^{\circ} \mathrm{C}$ incubator with $5 \% \mathrm{CO}_{2}$. Use these chambers immediately or store in the incubator for future use (two weeks).

5. Count the cell density of the differentiated HL60 cells with a hemocytometer. NOTE: The cell density often reaches about $1.0 \times 10^{6} \mathrm{cells} / \mathrm{ml}$. Cell density greater than $3.0 \times 10^{6} \mathrm{cells} / \mathrm{ml}$ usually gives undesirable transfection efficiency. As HL60 cells are suspension cells, no resuspension is required.

6. Centrifuge the cells at $200 \times \mathrm{g}$ for $5 \mathrm{~min}$ at RT. Remove the supernatant and resuspend $2 \times 10^{6} \mathrm{HL} 60$ cells in $100 \mu \mathrm{l}$ of transfection reagent.

7. Add $4 \mu \mathrm{g}$ of GFP-PKD1 plasmid ${ }^{9}$ into the $100 \mu \mathrm{l}$ mixture of cells and transfection reagents and mix gently and thoroughly. Add the mixture into the cuvette provided.

8. Select the manufacturer's pre-set program for the human leukocyte cell line and perform the electroporation. NOTE: Further information is available from the manufacturer's website.

9. After electroporation, immediately and gently add pre-warmed $500 \mu \mathrm{l} \mathrm{RPMI} 6140$ electroporation recovery medium to the cells, and gently transfer the cells from the cuvette to a $1.6 \mathrm{ml}$ tube with the plastic pipettes provided.

10. Incubate the cells for $30 \mathrm{~min}$ in a humidified $37^{\circ} \mathrm{C}$ incubator with $5 \% \mathrm{CO}_{2}$. Seed $100 \mu \mathrm{l}$ or $500 \mu \mathrm{l}$ of cells into one well of a 4 -well or a 1 -well chamber, respectively.

11. Add RPMI 1640 culture medium to a final volume of $1 \mathrm{ml}$ or $4 \mathrm{ml}$ to that same well of a 4-well or a 1-well chamber, respectively. Incubate the cells in a humidified $37{ }^{\circ} \mathrm{C}$ incubator with $5 \% \mathrm{CO}_{2}$ for $3 \mathrm{hr}$. Pre-warm RPMI 1640 starving medium to $37^{\circ} \mathrm{C}$.

12. Using a $1 \mathrm{ml}$ pipette, gently remove $0.8 \mathrm{ml}$ or $3 \mathrm{ml}$ of RPMl 1640 culture medium from the well of a 4 -well chamber or a 1 -well chamber, respectively. Be gentle and avoid sucking away adhering HL60 cells.

NOTE: HL60 cells grow and are differentiated in suspension media. After being differentiated or with certain treatments, HL60 cells adhere to the substratum coated with polylysine, collagen, gelatin, or fibronectin.

13. Add $0.8 \mathrm{ml}$ or $3 \mathrm{ml}$ of RPMl 1640 starving medium into the well of a 4-well or 1-well chamber, respectively. Wait $1 \mathrm{hr}$. 
NOTE: At this point the cells are ready for imaging experiments ${ }^{9}$.

\section{Monitoring GPCR-mediated Membrane Translocation of PKD1 by Multi-channel Fluorescent Microscopy}

1. Prepare a fresh mixture of $100 \mathrm{nM}$ fMLP and $1 \mu \mathrm{g} / \mathrm{ml}$ Alexa 594 in RPMl 1640 starving medium as stimuli. NOTE: The mixture of fMLP and Alexa 594 needs to be freshly made to ensure maximum efficacy.

2. Mount one 4-well chamber seeded with cells over a 40X oil lens.

3. Set the Fluorescent Microscope in the Multichannel Configuration as Follows:

1. Set the green emission channel $(500-530 \mathrm{~nm})$ for GFP-tagged PKD1, red emission channel (580-620 nm) for chemoattractant fMLP (Alexa 594), and transmitted light channel for identifying adhering HL60 cells.

2. Start "live" acquisition and optimize the acquisition conditions of the three channels:

3. Fine-tune between intensity and laser power for all three channels to minimize photobleach for a longer period of imaging.

4. If necessary, average every 2 to 4 frames to decrease the background noise ratio for better quality of images. Use a 1 sec interval.

4. Search for adhering cells that express strong PKD1-GFP in the view of the GFP and DIC (differential interference contrast) channel. Adhering cells are morphologically flat and do not move rapidly.

5. After identifying adhering HL60 cells that highly express GFP-PKD1, optimize the acquisition condition by lowering the laser power while increasing the detector gain for each channel to decrease the photobleach for long-term imaging (Figure 3A).

6. Take up $100 \mu \mathrm{l}$ of fMLP/Alexa 594 solution with a $200 \mu \mathrm{l}$ pipetter and set aside. Select the "time-lapse acquisition" and set the intervals to 2 sec and the number of frames to acquire to 100.

NOTE: the intervals and number of frames to acquire depend on the purpose of experiments. 1-2 sec intervals and 100 frames are often used for imaging experiments, as in Figure 3. For slowly migrating cells, longer intervals are necessary for long-term imaging. In contrast, for rapidly migrating cells, shorter intervals are required for observing the continuous details of the cell migration.

7. Carefully take off the lid of a 4-well chamber so that the position of the chamber is not changed. Start acquisition immediately and acquire $3-5$ images of the unstimulated cells while positioning the $200 \mu \mathrm{l}$ pipetter directly over the cells using the spot of laser light as a guide.

8. Pipette the fMLP/Alexa 594 mixture over the cells being imaged with a quick and even flow and finish the complete set of time-lapse acquisition. Name and save the data that will be subjected to further data analysis.

\section{Imaging Chemotaxing Cells in Visible and Controllable Chemo-attractant Stimuli}

1. Using a $10 \mu \mathrm{l}$ micropipetter and injection tips, backfill a micropipette with $30 \mu \mathrm{l}$ freshly prepared mixture of fMLP and Alexa594.

2. Attach the micropipette to a micropipette holder mounted on top of a microscope stage and connect the tubing to the pressure supply unit, an apparatus that provides a constant pressure to release fMLP/Alexa594 solution from the micropipette to generate a steady gradient.

3. Turn on the pressure supply and set the compensation pressure $(\mathrm{Pc})$ to $70 \mathrm{hPa}$.

4. Mount a 1-well chambered coverslip seeded with HL60 cells expressing PKD1-GFP over a 40X oil lens on a confocal microscope or its equivalent fluorescent microscope.

5. Set acquisition in channel mode in the following configuration: green channel $(500-530 \mathrm{~nm})$ for GFP-tagged PKD1; red channel (580-620 nm) for chemoattractant fMLP (Alexa594); and transmitted light channel for identifying adhering HL60 cells.

6. Start "live" acquisition and optimize the acquisition conditions for the three channels: adjust the intensities of each channel for quantitative analysis of imaging data; fine-tune between intensity and laser power for all three channels to obtain images with the best quality and minimize the photo-bleach. If necessary, average every 2 to 4 frames for better image quality. We usually use a $1 \mathrm{sec}$ interval.

7. Start "live" acquisition and search for adhering cells that express strong PKD1-GFP in the view of the GFP and transmitted light channels. Using bright-field optics, center the micropipette in the center field of view (Figure 4A) in order to visualize the fMLP gradient and the adhering HL60 cells expressing PKD1-GFP.

8. Select time-lapse acquisition and set the intervals to $2 \mathrm{sec}$ intervals and the number of frames to acquire to 100 . NOTE: After collecting each time-lapse series, name and save the data that will be subjected to further data analysis.

Simultaneous imaging of chemotaxis of multiple HL60 cells using cell mobility analysis device

Based on the principle of microfluidics ${ }^{16}$, the manufacturer has provided simulated profiles of gradients: a gradient is generated within 1 min, stabilized within $5 \mathrm{~min}$, and maintained over $2 \mathrm{hr}$. The highly predictable profiles of the stable gradients generated by microfluidics allow multiple chemotaxis assays to be carried out simultaneously. In the present study, we observed three simultaneous chemotaxis assays (Figure 2A and Movie 1). We found that HL60 cells started chemotaxing immediately after the chemoattractant was injected into the well of the chemoattractant, and kept chemotaxing in a straight path for the following $60 \mathrm{~min}$, consistent with the simulation results for gradient stability. Tracing the travel path and morphology of the cells allows quantitative measurements and subsequent comparison of the chemotaxis behaviors using a chemotaxis index that includes total path length, speed, directionality, and roundness of the cells (Figure 2B). Total path length is the sum of the lengths of the line segments connecting the centroids of the path. Speed is obtained by dividing the total path length by the time. Directionality is measured upward and is defined as: ( $Y$ coordinate of the end of the path minus $Y$ coordinate of the beginning) divided by total path length. This gives 1.0 for an object moving directly upward. The roundness of the cell is a measure (in percent) of how efficiently a given amount of perimeter encloses area. A circle has the largest area for any given perimeter and has a roundness parameter of $100 \%$. A straight line encloses no area and has a roundness parameter of $0 \%$. We show the quantitatively measured chemotaxis behavior as described by the selected chemotaxis parameters (Figure 2C).

Monitoring PKD subcellular localization in HL60 cells under a spatiotemporally visible and controllable fMLP stimulus 
It is a great technical advance to apply fluorescently labeled and controllable chemoattractant stimulation to an experimental system. Historically, we have applied either homogeneous (also called uniform) stimulation or gradient stimulation to observe cell response and behaviors. However, "blind" stimulation not only provides no spatiotemporal information on how the stimulus reaches the cells, but also casts doubt on any "abnormal" observations of cell response to stimulation, simply because we do not see the stimulus. We have previously shown that fluorescent dye (Alexa594) can be applied with chemoattractant to establish a linear relationship between chemoattractant concentration and monitored fluorescent dye intensity ${ }^{15}$. With an acquisition configuration of green fluorescent protein (GFP), a red emission of fluorescent dye (Alexa594), and transmitted light, we are able to monitor the adhering cells, the application of the stimulus, and the cell response to the stimulus (Figure 3A). Protein kinase $\mathrm{D}$ is a family of serine/threonine kinases that play essential roles in directed cell migration ${ }^{9,17}$. In response to uniformly applied fMLP (red) stimulation, HL60 cells mediate a robust membrane translocation of GFP-tagged protein kinase D1 (green) (Figure 3B and Movie 2). In an fMLP gradient (red) (Figure 4A), HL60 cells actively recruit PKD1 to the leading edge (Figure 4B and Movie 3). A close comparison of the subcellular localization of GFP in the protrusion of the leading edge indicates that PKD1 localizes at the rear of the leading edge (Figure 4C).

A

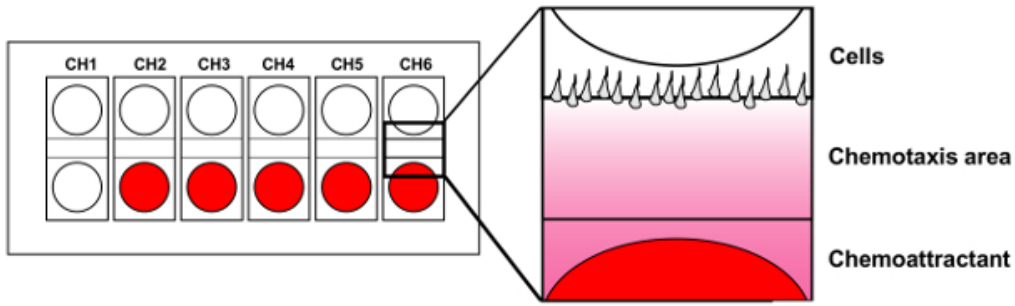

B
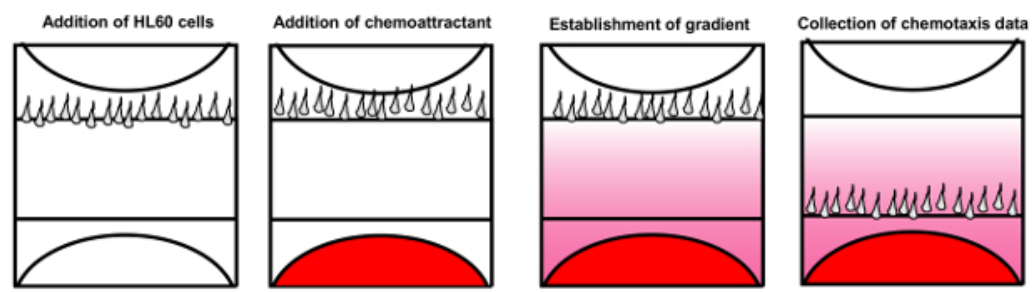

Figure 1. Cell mobility analysis device allows up to 6 simultaneous chemotaxis assays. (A) Scheme shows the design of a cell mobility analysis device chip for simultaneous monitoring of 6 independent chemotaxis assays. Red shows chemoattractant added to the wells. (B) Introduction of HL60 cells to the wells of cells while the chemoattractant diffuses to establish a steady fMLP gradient. Please click here to view a larger version of this figure.
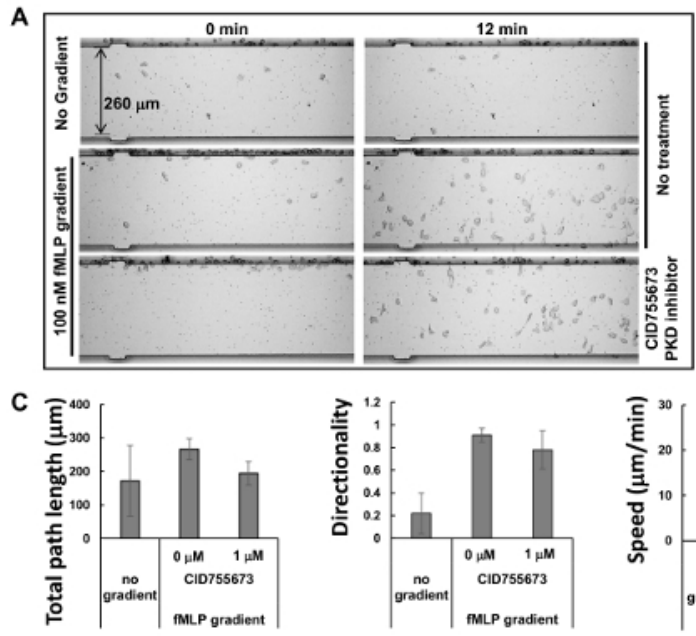
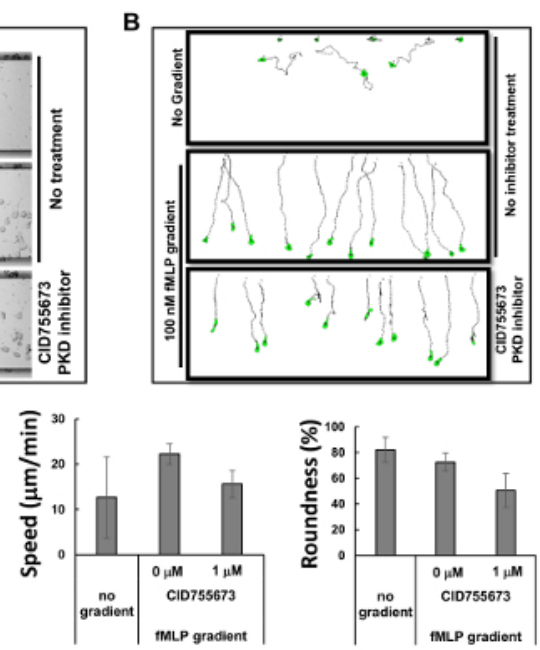

Figure 2. Simultaneous monitoring of multiple chemotaxis assays with HL60 cells. (A) Montage shows images of cell mobility analysis device chemotaxis assay to examine the inhibitory effects of PKD-specific inhibitors on chemotaxis at times of 0 and 12 min after gradient application. Chemotactic HL60 cells were pre-treated with PKD inhibitor $1 \mu \mathrm{M}$ CID755673 for $30 \mathrm{~min}$. HL60 cells with or without the treatment of PKD inhibitor were allowed to chemotax in either RPMI1640 starving medium or $100 \mathrm{nM}$ fMLP gradients for 12 min. (B) Scheme shows the travel path length and morphology of traced HL60 cells. (C) Quantification of chemotaxis as total path length, speed, directionality, and roundness. Mean \pm SD is shown; $n=10,12$, or 11 for no gradient, fMLP gradient without CID755673 treatment, and fMLP treatment with CID755673 treatment, respectively. Please click here to view a larger version of this figure. 
A

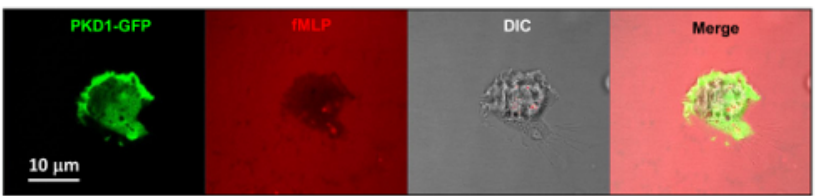

B
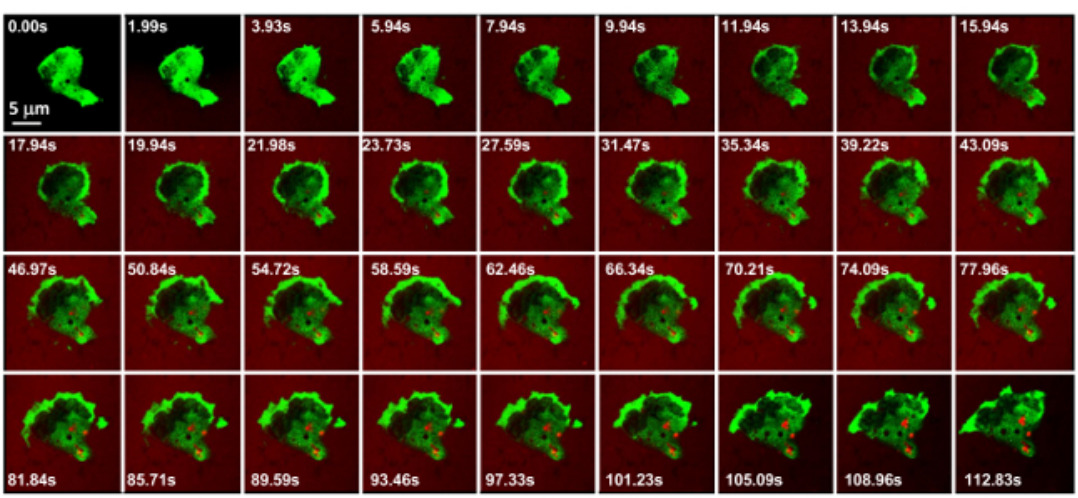

Figure 3. GPCR-mediated robust membrane translocation of PKD1 in response to uniformly applied fMLP stimuli. (A) Multichannel monitoring of PKD1-GFP (green), chemoattractant (1 $\mu \mathrm{M}$ fMLP mixed with $0.1 \mu \mathrm{g} / \mathrm{ml}$ fluorescent dye Alexa 594 , red), and DIC (differential interference contrast) to identify the adhering HL60 cells in a well of a 4well chamber coated with $0.2 \%$ gelatin in RPMI 1640 medium. Scale bar $=10 \mu \mathrm{m}$. (B) Montage shows that uniformly applied fMLP (red) induces robust membrane translocation of PKD1-GFP (green). Please click here to view a larger version of this figure.
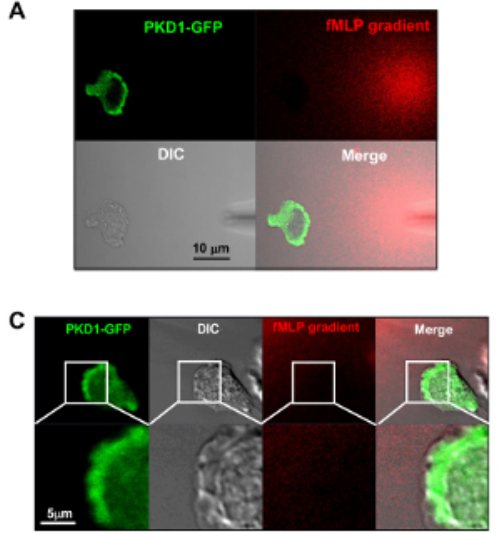

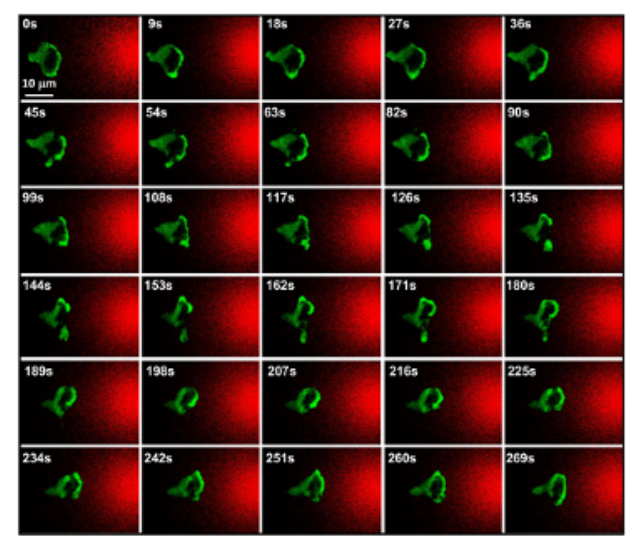

Figure 4. Leading edge localization of PKD1 in chemotaxing HL60 cells. (A) Channel mode acquisition configuration facilitates the visualization of the fMLP gradient and the spatiotemporal dynamics of PKD1. In A-C, HL60 cells transiently expressed GFP-tagged PKD1; to visualize the fMLP gradient generated from a micropipette (DIC), $100 \mathrm{nM}$ fMLP (Red) was mixed with $0.1 \mu \mathrm{g} / \mathrm{ml}$ fluorescent dye Alexa 594 . (B) Enriched localization of PKD1 at the leading edge of the chemotaxing cell. Scale bar $=10 \mu \mathrm{m}$. (C) Merged images show that PKD1 localizes at the rear of the leading edge in HL60 cells. Green shows PKD1 cellular localization, and the DIC image shows the protruding area of the leading edge. Scale bar $=5 \mu \mathrm{m}$. Please click here to view a larger version of this figure.

\section{Discussion}

In the present study, we show two examples of chemotaxis assays: first, simultaneous monitoring of multiple chemotaxis assays by the cell mobility analysis device; and second, visualization of the chemoattractant gradient and the spatiotemporal dynamics of signaling events in the same cells in real time.

Cell mobility analysis device for multiple simultaneous chemotaxis assays

In the present study, we introduced a detailed protocol to perform multiple simultaneous chemotaxis assays using a cell mobility analysis device. This device allows user to observe cellular chemotaxis behavior with a 10X objective lens in conventional bright-field observation. Because of the diffusion-dominant characteristics of the fluid flow, the cell mobility analysis device generates highly predictable, stable gradients and allows up to six chemotaxis assays to be carried out simultaneously. The critical steps of the protocol are to obtain reliable gradients and to align the cells on the terrace line. The user should strictly follow the manufacturer's instructions for holder assembly and injection of chemoattractants and cells. Detailed instructions are also available online. Compared to alternative chemotaxis methods ${ }^{11-13,15}$, this device significantly improves the reliability and efficiency of chemotaxis assays. Four sizes of cell mobility analysis device chip in sizes of $4,5,6$, and $8 \mu \mathrm{m}$ are available to accommodate different types and sizes of cells. We found that a 4 or $5 \mu \mathrm{m}$ cell mobility analysis device chip is suitable for the HL60 and $D$. 
discoideum cells, which are about 10-15 $\mu \mathrm{m}$ in diameter. However, one limitation is that this device is not suitable for all types of cells. We had little success using the cell mobility analysis device chemotaxis assay with Raw267.4 cells. The reason may be that Raw267.4 cells migrate too slowly. The time required for efficient chemotaxis of Raw267.4 cells might be much longer than the time a gradient is maintained by the device. Instead, a transwell migration assay worked well for Raw267.4 cells ${ }^{9}$. Another limitation is that fluorescent observation is not possible with the current device. A future direction is to monitor fluorescent imaging with higher magnification. This is possible with the improved cell mobility analysis device, which is equipped with fluorescent detection and a 100X objective lens. In addition, the number of simultaneous assays is also increased to 12. All these improvements facilitate the enhanced throughput of chemotaxis assays and the observation of subcellular dynamics in migrating cells.

High transfection efficiency of HL60 cells to express fluorescent protein-tagged protein

HL60 cells are an actively dividing leukemia cell line and grow in suspension. As previously reported ${ }^{18-20}$, HL60 cells are resistant to gene transfer. Both lipid and electroporation gene transfer have been tested, and higher transfection efficiency was obtained with electroporation, as described in detail in section 4. Obtaining high viability after electroporation is critical for obtaining high transfection efficiency because of the severe damage that results from electroporation. Subsequently, thorough and gentle cell handling are required, especially after electroporation. All media have to be pre-warmed and gently added to the cells in any steps after electroporation. It is also critical to minimize the exposure time of cells to the electroporation reagent. To avoid cell death, after electroporation, RPMI 1640 electroporation recovery medium must be added to the cells immediately. We found that $20 \%$ FBS in the recovery medium gives a much higher cell recovery rate than $10 \%$ FBS. After electroporation, incubation in RPMI 1640 electroporation recovery medium for 30 min is critical for greater viability and transfection efficiency. To further increase transfection efficiency, we also used $4 \mu \mathrm{g}$ plasmid DNA per transfection, which is almost the twice amount of plasmid recommended by the manufacturer.

There are two major limitations on electroporation transfection: the transiency of the protein expression and the cell number limit $\left(2 \times 10^{6}\right.$ cells per transfection). In undifferentiated cells, expression is detectable only during the first couple of cell divisions, since the vector plasmid is diluted by half after each cell division. For The differentiated HL60 cells survive no more than $48 \mathrm{hr}$. As a result, any experiment requires fresh transfections $6 \mathrm{hr}$ prior to the experiment. The cell number for one electroporation merely meets the minimum requirement for any biochemical assays. For the purpose of repetitive use or large quantity, it is strongly recommended that an undifferentiated HL60 cell line be established that stably expresses the protein of interest which has been tagged with a fluorescent protein by a viral vector, if a viral vector is available or can be constructed.

\section{Disclosures}

The authors declare no competing financial interest for this work.

\section{Acknowledgements}

This work is supported by the intramural fund of NIAID, NIH.

\section{References}

1. Pocha, S. M., \& Montell, D. J. Cellular and molecular mechanisms of single and collective cell migrations in Drosophila: themes and variations. Annual review of genetics. 48, 295-318 (2014).

2. Wen, Z., \& Zheng, J. Q. Directional guidance of nerve growth cones. Current opinion in neurobiology. 16, 52-58 (2006).

3. Bravo-Cordero, J. J., Hodgson, L., \& Condeelis, J. Directed cell invasion and migration during metastasis. Current opinion in cell biology. 24, 277-283 (2012).

4. Zigmond, S. H. Chemotaxis by polymorphonuclear leukocytes. The Journal of cell biology. 77, 269-287 (1978).

5. Jin, T., Xu, X., \& Hereld, D. Chemotaxis, chemokine receptors and human disease. Cytokine. 44, 1-8 (2008).

6. Dong, X. et al. P-Rex1 is a primary Rac2 guanine nucleotide exchange factor in mouse neutrophils. Current biology : CB 15, 1874-1879 (2005).

7. $\mathrm{Li}, \mathrm{Z}$. et al. Directional sensing requires $\mathrm{G}$ beta gamma-mediated PAK1 and PIX alpha-dependent activation of Cdc42. Cell 114, 215-227 (2003).

8. Van Haastert, P. J., \& Devreotes, P. N. Chemotaxis: signalling the way forward. Nature reviews. Molecular cell biology. 5, 626-634 (2004).

9. Xu, X. et al. GPCR-Mediated PLCbetagamma/PKCbeta/PKD Signaling Pathway Regulates the Cofilin Phosphatase Slingshot 2 in Neutrophil Chemotaxis. Mol Biol Cell (2015).

10. Boyden, S. The chemotactic effect of mixtures of antibody and antigen on polymorphonuclear leucocytes. The Journal of experimental medicine. 115, 453-466 (1962).

11. Zigmond, S. H. Ability of polymorphonuclear leukocytes to orient in gradients of chemotactic factors. The Journal of cell biology. 75 , 606-616 (1977).

12. Zicha, D., Dunn, G. A., \& Brown, A. F. A new direct-viewing chemotaxis chamber. Journal of cell science. 99 ( Pt 4), $769-775$ (1991).

13. Muinonen-Martin, A. J., Veltman, D. M., Kalna, G., \& Insall, R. H. An improved chamber for direct visualisation of chemotaxis. PloS one. 5, e15309 (2010)

14. Bozzaro, S., Fisher, P. R., Loomis, W., Satir, P., \& Segall, J. E. Guenther Gerisch and Dictyostelium, the microbial model for ameboid motility and multicellular morphogenesis. Trends in cell biology. 14, 585-588 (2004).

15. Xu, X., \& Jin, T. Imaging G-protein coupled receptor (GPCR)-mediated signaling events that control chemotaxis of Dictyostelium discoideum. Journal of visualized experiments : JoVE. (2011).

16. Lin, F. et al. Generation of dynamic temporal and spatial concentration gradients using microfluidic devices. Lab Chip 4, 164-167 (2004). 
17. Eiseler, T. et al. Protein kinase D1 regulates cofilin-mediated F-actin reorganization and cell motility through slingshot. Nature cell biology 11, 545-556 (2009).

18. Roddie, P. H., Paterson, T., \& Turner, M. L. Gene transfer to primary acute myeloid leukaemia blasts and myeloid leukaemia cell lines. Cytokines Cell Mol Ther. 6, 127-134 (2000).

19. Schakowski, F. et al. Novel non-viral method for transfection of primary leukemia cells and cell lines. Genet Vaccines Ther 2, 1 (2004).

20. Uchida, E., Mizuguchi, H., Ishii-Watabe, A., \& Hayakawa, T. Comparison of the efficiency and safety of non-viral vector-mediated gene transfer into a wide range of human cells. Biol Pharm Bull. 25, 891-897 (2002). 